\title{
Adaptive Management of Cognitive Radio Networks Employing Femtocells
}

\author{
Anwer Al-Dulaimi, IEEE, Member, Alagan Anpalagan, IEEE, Senior Member, Saba Al-Ruabye, IEEE, Member, \\ and Qiang Ni, IEEE, Senior Member
}

\begin{abstract}
Network planning and management are challenging issues in a two-tier network. Tailoring to cognitive radio networks, network operations and transmissions becomes more challenging due to the dynamic spectrum availability. This paper proposes an adaptive network management system that provides switching between different cognitive radio network management structures in response to the spectrum availability and changes in the service time required for the radio access. The considered network management system includes conventional macrocell-only structure, and centralized/distributed structures overlaid with femtocells. Furthermore, analytical expressions of per-tier successful connection probability and throughput are provided to characterize the network performance for different network managements. Spectrum access in dynamic radio environments is formulated according to the quality of service $(\mathrm{QoS})$ constraint that is related to the connection probability and outage probability. Results show that the proposed intelligent network management system improves the maximum capacity and reduces the number of blocked connections by adapting between various network managements in response to free spectrum transmission slots. A road map for the deployment and management of cognitive macro/femto networks is also presented.
\end{abstract}

Index Terms - Cognitive radio, femtocell, network management, spectrum access, throughput

\section{INTRODUCTION}

$\mathrm{E}$ stablishing a self-organizing cognitive radio network (CRN) requires the coordination of various network functionalities such as channel access, topology management, and message control, in addition to normal network operations. For wireless network architectures operating with multi-channels, it is important to set up the most appropriate network model to ensure flexible and effective use of the spectrum resources [1], [2]. In order to develop efficient network planning, deployment, and management, it is necessary to identify the core functions required for supporting network operations. Therefore, a block diagram of the main cognitive radio network functionalities is shown in Fig. 1, based on an enhanced telecom operations map (eTOM) business process framework [3]. The prominent components are:

Network Considerations: This component generates a network plan as an output. This plan includes high-level configuration information for all the network components, including information about the available resources such as radios, frequency spectrum, etc.
Operations Management: This component is responsible for implementing the required policy for the network adaptation functionality, as well as setting profiles for various interfaces.

Performance and Configuration Management: These critical functions are responsible for quality of service (QoS) assurance and providing admission control in order to prioritize traffic.

Cell-Site Management: This component computes the most appropriate transmission platform for different network sites, allocates resources, manages configurations, and interfaces with the end users.

The shadowed blocks in Fig. 1 are the functions of interest when managing multi-tier network systems or assigning resources between the subcell domains considered in this paper.

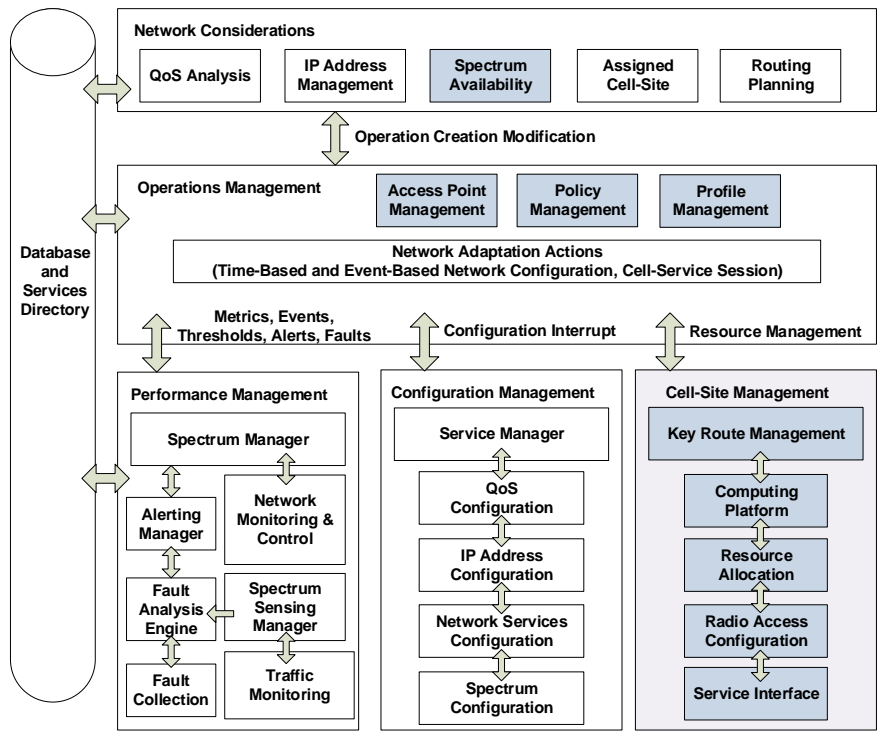

Fig. 1. Operation functions for management and service access in cognitive radio networks.

In this paper, we investigate and derive the basic performance requirements for deploying a scalable and flexible architecture that consists of a cognitive radio network. Based on these requirements, we analyze a hybrid structure composed of conventional, centralized, and distributed network management. The main components of this structure are: (i) macrocell and femtocell domains for radio access communications, and (ii) a spectrum broker for optimization and network adaptation management. In order to evaluate and validate the feasibility of the proposed structure, we develop a 
dynamic wireless spectrum profile followed by a mathematical model for a small-sized two-tier macro-to-femto network. The performance of each network model of centralized and distributed planning is examined and compared to the macro-only network model to validate deployment conditions. The spectrum broker reacts to the changes in site domains, choosing to which network management they should assign operations and users, therefore acting as a dynamic self-organized system that adapts infrastructure according to the operating wireless environment. The contributions of this paper are arranged into stages in order to develop a large-scale network system model, as shown in Fig. 2.

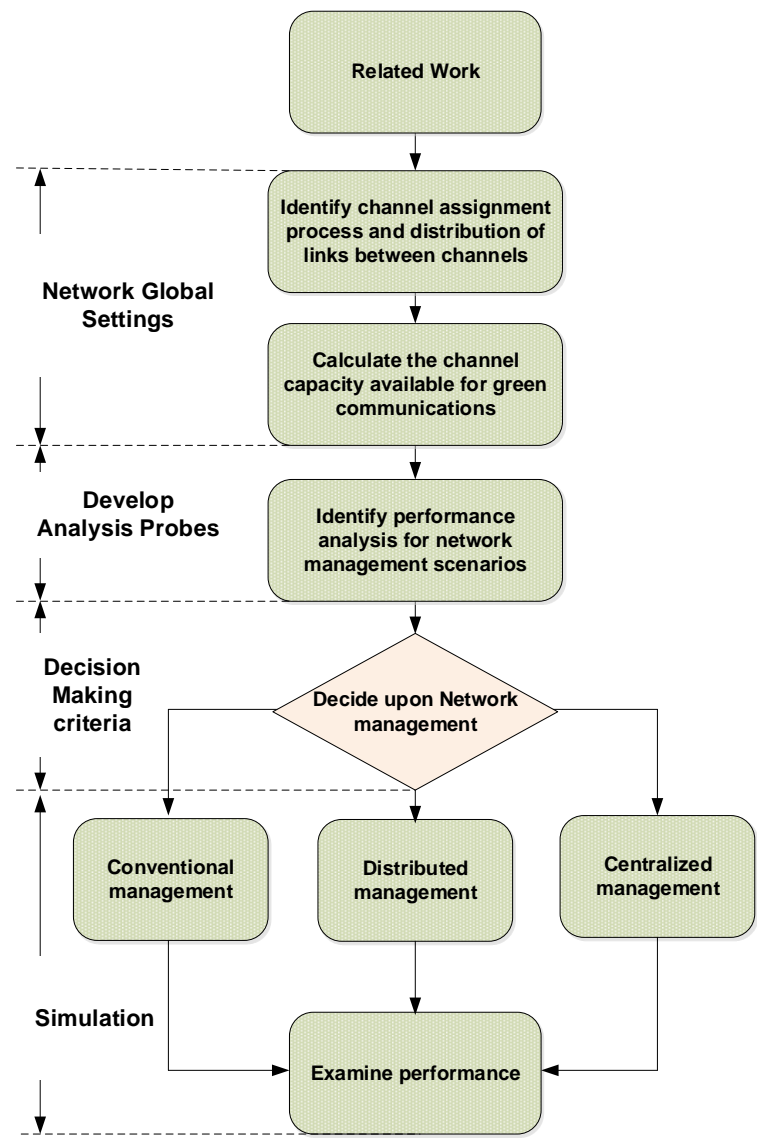

Fig. 2. Overview of contents of this paper.

This study provides an insight into how rational users can be distributed among existing access solutions (centralized networks versus distributed networks), i.e., the criteria of choosing between different structures. We adopt a femtocell-aided macrocell network using stochastic geometric methods (as in [4]-[8]) to study the steady-state performance of each network management model, focusing on the number of blocked connections as a quality of service (QoS) metric. In order to formulate the selection process for network structures, we consider a joint subchannel scheme in which the whole spectrum is shared by both tiers, as well as a disjoint subchannel scheme, whereby disjoint sets of subchannels are assigned to each tier. First, we provide analytical expressions for the per-tier successful connection probability and network throughput as a means to characterize the performance of different network management structures. Second, we formulate the intelligent access to the spectrum subject to the QoS constraint, and expressed in terms of minimum per-tier successful connection probability and outage capacity. We also provide useful insights into the network management system by developing selected metrics that can improve the network performance.

The remainder of this paper is organized as follows. Related work is reviewed in Section II. The network model for the proposed multi-level control and network selection are described in Section III. The conventional, centralized, and distributed models of network management are discussed in Sections IV, V, and VI, respectively. The network adaptation model for selecting network management is presented in Section VII. Simulation settings and results are discussed in Section VIII, whereas Section IX concludes the paper.

\section{RELATEd WORK}

Among a few types of research proposals pertaining to the network management process, we discuss the major works related to system management and resource allocation between macro- and femtocell units. The analytical model for multicell systems in [4] studied the effect of spectrum allocation in two-tier networks by considering joint subchannel allocation, in which the whole spectrum is shared by both tiers, as well as disjoint subchannel allocation, wherein disjoint sets of subchannels are assigned to both tiers. Although, joint subchannel allocation may be sensible in dense networks, it is not clear whether disjoint subchannel allocation would be necessary in lightly loaded network sites where interference incurred through subchannel sharing can be tolerated. However, there is no association between channel allocation and network structure.

The resource allocation in open orthogonal frequency division multiple access (OFDMA) femtocell networks can be used to improve the QoS for the neighboring macrocell users. Using OFDMA, the authors in [5] proposed a multi-access technique that allocates different users to different groups of orthogonal subchannels exploiting channel variations in both frequency and time domains. The spectrum-sharing scheme proposed in [6] achieved high data rates for macrocell-femtocell networks, and for the femtocell networks, by improving the spatial reuse gain. The authors in [7] proposed an analytical approach to improve the spectrum sharing in macrocell-femtocell networks. The studied system model exploits a new spectrum swapping access strategy that improves macrocellular performance as being the main transmission unit in any network site. These solutions propose only physical layer improvements with no consideration to the network management and resource allocations between network tires.

The work in [8] developed a tractable, flexible, and accurate model for a downlink heterogeneous cellular network as a solution for two-tier networks. Even with a Poisson point process model, the outcome of this research is about as accurate as the standard grid model, when compared to an actual network. Most importantly, the authors mentioned that for a 
network model to be applicable and accurate, it should consider using the mathematical tools of stochastic geometry to bear on the problem of base stations locations. This helps to investigate the fundamental performance of wireless networks.

In [9], the authors investigated the downlink spectrum-sharing problem while applying cognitive radio technology to femtocell networks. However, the given solution did not investigate the overall network operation scenarios, nor analyze a dynamic profile for the spectrum availability. Moreover, the cross-tier interference avoidance strategy, which was developed in [10], used macrocell uplink interference in two-tier OFDMA networks to derive the distribution of macrocell uplink interference, including intercell and cross-tier interference. These analyses were conducted by assuming a homogeneous spatial Poisson point process for femtocell distribution across macrocell site.

Different from the available literature, this paper addresses the adaptive management of the cognitive radio network as an approach to solve the problem of capacity maximization and to reduce the number of blocked connections. The paper proposes different network management structures that can be used to efficiently utilize the available resources for the same traffic load profile and different free spectrum transmission slots. The goal is to identify the optimal scales for cognitive radio networks operating with low and high numbers of free channels.

\section{SYSTEM MODEL}

There is a considerable amount of unused spectrum holes that temporarily become available in the licensed spectrum band [11]. Therefore, cognitive radio networks are anticipated to utilize these transmission opportunities through cognitive communication techniques. As a general framework, we propose, in Fig. 3, a heterogeneous network (HetNet) architecture of a cognitive network that coexists with the primary user (PR) network on site. The cognitive network is composed of one macrocell overlaid by femtocell domains.

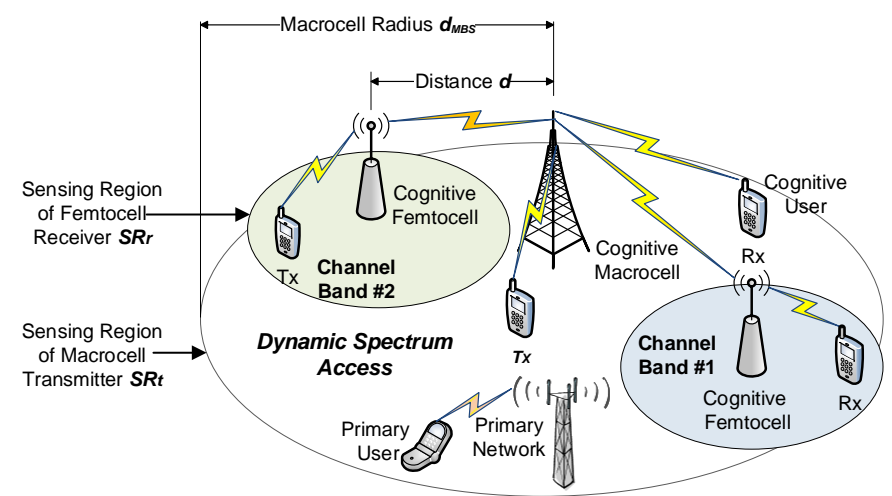

Fig. 3. Coexistence of cognitive and primary networks. The underlying architecture of a cognitive network is a macrocell employing femtocell domains.

TABLE I

SYSTEM MYTHOLOGY $e_{i j} \quad$ Link formed when node $i$ transmits to node $j$

$E_{t} \quad$ Set of active links in a certain tier $t$, where $t=\{1,2\}$

$\operatorname{SIR}\left(e_{i j}, t\right)$ Signal-to-interference ratio at the edge $e_{i j}$ in tier $t$

$\gamma_{t h} \quad$ Threshold in tier $t$, same for both macro and femto tiers

$e_{i} \quad$ Transmitter link of node $i$

$e_{i j} \quad$ Receiver link of node $j$

$R_{\max } \quad$ Maximum transmission radius in the cell (macro or femto)

$\delta_{t h} \quad$ Step distance in any tier $t$, same for both macro and femto tiers

$S_{i} \quad$ Set of femtocell-tier channels assigned to link $e_{\mathrm{i}}$

$\lambda \quad$ Density of licensed users per unit area

$n \quad$ Number of licensed users in area $A$

$d_{M B S} \quad$ Macrocell radius in meters

$d \quad$ Femtocell distance from the macrocell unit in meters

$C \quad$ Cognitive channel capacity

$X \quad$ Number of cognitive network users

$\mathrm{P} \quad$ Maximum transmitted power from cognitive macrocell

$N \quad$ Number of free channels

$L \quad$ Number of occupied channels

$P_{\text {block }} \quad$ Probability of blocked connections

$k \quad$ Number of non-overlapping CAB blocks

$m \quad$ Number of service managers

$l_{m ; i} \quad$ Size of the spectrum block in frequency range $(b ; e)$

$s_{G} \quad$ Minimum guard band

$I_{C A B} \quad$ Minimum CAB size

$\lambda_{\mathrm{h}} \quad$ Arrival rates for a high-priority unlicensed user

$\lambda_{1} \quad$ Arrival rates for a low-priority unlicensed user

$\mu \quad$ Mean connection-holding time during call approval

$\vartheta \quad$ Network throughput

$a_{x} \quad$ Network load

$v_{x} \quad$ Total number of cells in the network site

$\mathrm{o}_{w}^{\prime} \quad$ Octet data that has a number of $\mathrm{W}-$ th frames

B $\quad$ 802.11 PHY mode (chosen as 7)

$g$ Average SNR per symbol at the 802.11 receiver

$\rho \quad$ 802.11a PLCP preamble duration $(16 \mu \mathrm{s})$

$\sigma \quad$ 802.11a PLCP signal field duration $(4 \mu \mathrm{s})$

$\varphi \quad$ 802.11a OFDM symbol interval $(4 \mu \mathrm{s})$

$\mathrm{N}_{\text {cbps }}($ ( $)$ Number of coded bits per sample $\left(\mathrm{N}_{\text {cbps }}(7)=192\right)$

$\mathrm{T}_{\text {data }}\left(o_{w}^{\prime}\right.$, E) Transmission duration of the data frame

$\mathrm{T}_{\text {bar }}$ (Б) Transmission duration of the chunk Ack Request frame

$\mathrm{T}_{\mathrm{ba}}$ (Б) Transmission duration of the Block Ack frame

$\mathrm{T}_{\text {cfpoll }}$ (Б) Transmission duration of the CF-poll frame

$\mathrm{P}_{g, \operatorname{trans}(\mathrm{y})}\left(\sigma^{\prime}, g\right.$, b) Probability of successful chunk transmission

$\mathrm{P}_{\mathrm{e}, \text { data }}\left(\sigma^{\prime}, g, \mathrm{~B}\right)$ Error probability of data

$P_{e, b a r}(g, m)$ Error probability of chunk Ack

$P_{e, b a}(g, m)$ Error probability of chunk Ack frame

$\mathrm{N}_{\text {burst }} \quad$ Number of burst MAC service data units (MSDUs)

$\mathrm{N}_{\text {MPDU }}$ Number of MAC protocol data units (MPDUs) per one MSDU

$\mathrm{N}_{\text {burst(y) }}$ Number of MPDUs retransmitted at y transmissions

y Number of attempts to transmit $\mathrm{N}_{\text {burst(i) }}$ MSDUs

Q Octet long packet

$\partial_{\text {free }} \quad$ Free distance of the convolutional code mode Б

$\mathrm{a}_{\partial} \quad$ Total number of error events of weight $\mathrm{d}$

$\mathrm{P}_{\partial}(g)$ Probability of incorrect path at $\mathrm{d}$ from the correct path

$\mathrm{P}_{\mathrm{u}}^{(\mathrm{B})}(g) \quad$ First-event error probability

$\mathrm{P}_{\text {success }}(\sigma, g$, Б)Probability of a successful transmission

$\eta_{\max }$ Retry limit

$\mathrm{T}_{\mathrm{r}} \quad$ Response time

$\mathrm{t}_{\mathrm{SP}} \quad$ Computation time at the spectrum broker

$t_{a} \quad$ Time between the mobile user arrival at femtocell and SM updates $t_{N} \quad$ Overall network delay

The complexity of evaluating the performance of the network managements comes from the fact that it is necessary to develop many functions that characterize large-sized network operations. We start by identifying the channel assignment in cognitive two-tier system mode and the fairness of this assignment at the femtocell-tier, and then we model the 
cognitive radio network sensing functionality according to network size in order to calculate the capacity available for cognitive communication. The notions for the macro and femto cellular domains in Fig. 3 are given in Table I.

\section{A. Channel Assignment}

In order to perform successful connections, cognitive radio networks need to determine the best available transmission opportunities, and then configure operations. This procedure demands a flexible network management system to allow the adaptation of network structure, in response to the dynamic changes in the wireless environment. Therefore, channel assignment algorithms, which are used for frequency cellular networks, are not applicable for cognitive radio networks. The optimal channel assignment problem in cognitive radio networks must take into account factors such as resource allocation in multi-tier systems and multi-management network models.

In this subsection, we provide a global channel assignment model that is applicable to cognitive networks, whether they employ macrocells only or macro-to-femto management. In this way, the problem of channel assignment is extended further by considering the link quality constraints. The link quality constraints refer to the signal to noise (SNR) ratio or the distance ratio that is used to assign users to a certain channel at a certain tier. The communication between users is performed within any cell, i.e., node $i$ transmits to the base station, which in turn transmits to the receiver node $j$. The SNR constraint should be higher than a particular threshold $\gamma_{t h}$ in order to obtain a valid channel assignment scheme. If $E_{t}$ is the set of all links assigned to tier $t$, then channel assignment is said to satisfy the minimum SNR constraint if [12]:

$$
\operatorname{SNR}\left(e_{i j}, t\right) \geq \gamma_{t h}, t=\{1,2\}, \forall e_{i j} \in E_{t},
$$

We assume that the transmitting user has to establish a link first with the nearest base station and then with the call passed to the receiving user. For example, a link $e_{i}$ is assigned to tier $t$, i.e., $\forall e_{i j} \in E_{t}$, and it needs to satisfy the minimum distance ratio limit if [13]:

$$
d\left(T_{x}\left(e_{j}\right),\left(R_{x}\left(e_{i}\right)\right) \geq\left(1+\delta_{t h}\right) R_{\max },\right.
$$

where $R_{\text {max }}$ is the maximum transmission radius of the cell, $T_{x}\left(e_{j}\right)$ denotes the transmitter of link $e_{j}$, and $R_{x}\left(e_{i}\right)$ is the receiver of link $e_{i}$. That is, the distance between the receiver of the given link and the transmitters of other active links sharing the same tier $t$ should be larger by a factor of $\left(1+\delta_{t h}\right)$ compared to the maximum transmission radius of the cell.

We now provide the criteria for the channel distribution in the femtocell tier. Therefore, this channel modeling is only used by cognitive network management that employs femtocell systems. For the femtocell management, we do fair distribution of the available channels between femtocells in operation. The allocation of channels in the femtocell tier is subject to the management model used. To start relating the assignment of resources to network modeling scenarios, let $S_{i}$ be the set of subchannels assigned to link $e_{i}$ at the femtocell tier. Then $S_{i}$ can be given as:

$$
S_{i} \triangleq\left\{t, e_{i} \in E_{t}, \text { where } t=1\right\}
$$

Depending on the number of subchannels assigned to each link, channel assignment can be classified as unfair, justified, or fair. A channel assignment is called unfair if there is at least one link that is not assigned to any subchannel, i.e., $\exists i \in$ $\left\{1, \ldots,\left|E_{t}\right|\right\}$ such that $\left|S_{i}\right|=0$. Such an assignment could lead to loss of connectivity in a multihop network and should be avoided. To preserve connectivity, it is essential that $\left|S_{i}\right| \geq$ $1 \forall i \in\left\{1, \ldots,\left|E_{t}\right|\right\}$. A channel assignment that ensures connectivity is called a justified assignment. A channel assignment is called fair if $\left|S_{i}\right|=1 \forall i \in\left\{1, \ldots,\left|E_{t}\right|\right\}$ [11], [12].

\section{B. Cognitive Channel Capacity}

In this subsection, we identify the cognitive channel capacity probe that will be used in evaluating the performance of different cognitive network managements throughout this paper. Specifically, we propose to study the spectral efficiency by considering throughput analysis in each network tier. This sets the merit figures for the following investigations of cognitive radio network management scenarios. In this regard, the activity of primary users on each channel can be modeled as an on/off process where the durations of on and off periods are geometrically distributed over the network transmission domains. This means that the transmission opportunities exploited by the cognitive network are not identical. To analyze the throughput between cognitive macrocell and femtocell base stations shown in Fig. 3, it is assumed that the spatial distribution of the licensed users follows a Poisson distribution with the density of $\lambda$ licensed users per unit area. Hence, the probability of having $n$ licensed users in area $A$ is obtained from:

$$
\mathrm{P}_{\mathrm{r}}(n \text { users in } A)=P_{r}(\mathcal{F}(\mathrm{A})=\mathrm{n})=\frac{\mathrm{e}^{-\lambda A}(\lambda A)^{n}}{n !}
$$

In our study, we define two differently sized regions of sensing, one for the macrocell and one for the femtocell domains. To simplify the analysis, we assume that the macrocell base station acts as the transmitter for a circular macrocell with radius $d_{M B S}$ (where $d_{M B S}=R_{\max }$ ), while the cognitive femtocell acts as the receiver that is located at distance $d$ from the macrocell unit. Therefore, the probability that the macrocell detects an active primary user within its coverage area [14] is given by:

$$
\begin{gathered}
\mathrm{P}_{\mathrm{r}}\left(s_{t}=1\right)=P_{r}\left(\mathcal{F}\left(\mathrm{SR}_{\mathrm{t}}\right)=0\right)=P_{r}\left(\mathcal{F}\left(\pi\left(d_{M B S}\right)^{2}\right)=0\right) \\
=\mathrm{e}^{-\lambda \pi\left(d_{M B S}\right)^{2}} \\
\mathrm{P}_{\mathrm{r}}\left(s_{t}=s_{r}=1\right)=P_{r}\left(\mathcal{F}\left(\mathrm{SR}_{\mathrm{t}} \mathrm{USR} \mathrm{SR}_{\mathrm{r}}\right)=0\right) \\
\quad=\mathrm{e}^{-\lambda\left(2\left(d_{M B S}\right)^{2}\left(\pi-\cos ^{-1}\left(\frac{d}{2 d_{M B S}}\right)\right)+d \cdot d_{M B S} \sqrt{1-\frac{d^{2}}{4\left(d_{M B S}\right)^{2}}}\right)}
\end{gathered}
$$


Therefore, the capacity of the cognitive channel is related to the network size and it is fitted to the system model of Fig. 3, similar to [14] as given by:

$$
C=\mathrm{e}^{-\lambda\left(2\left(d_{M B S}\right)^{2}\left(\pi-\cos ^{-1}\left(\frac{d}{2 d_{M B S}}\right)\right)+d \cdot d_{M B S} \sqrt{1-\frac{d^{2}}{4\left(d_{M B S}\right)^{2}}}\right)}
$$

where $P$ is the maximum transmission power of the cognitive macrocell.

In this section, we provided the global settings used for the system evaluations throughout this paper. In the next sections, we study the conventional one-macro system management for cognitive radio networks and start developing approaches to measure its performance. This is followed by developing the performance characterization of the macro/femto centralized and distributed network management models.

\section{CONVEnTional Network Management}

The most popular and standard management of mobile networks is the macrocell model in which a macrocell unit performs mobile communications all over the cell site [15]. This type of management is also applicable in cognitive networks where a cognitive macrocell base station performs cognitive communication in coexistence with a primary network as shown in Fig. 4.

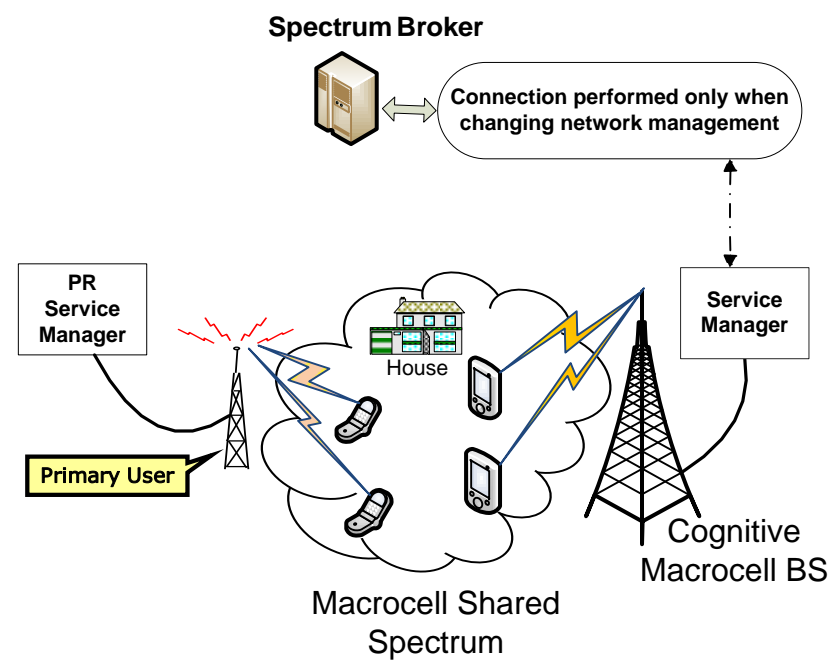

Fig. 4. Conventional network management.

The performance evaluation in this paper is based on examining the number of blocked connections and the throughput for each network management system. The development of a probe that measures the blocked connections is presented in the next section and given in (16). The network throughput can be calculated as:

$$
\vartheta=\frac{\text { Total transmitted packets }}{\text { Total polling time }}
$$

It is widely accepted that incorporating femtocells within macrocellular networks can significantly improve the network performance and spectrum utilization [16], [17]. However, to the best of our knowledge, we could not find any work in the literature that analyses the cognitive macro/femto system management or network structure solutions for cognitive communications. In the following sections, we propose the potential network management that allows efficient deployment of outdoor femtocells in cognitive macrocells using centralized and distributed management schemes. Also, we later propose an adaptive network management system that allows switching between different network management scenarios according to the spectrum availability and service time required for radio access.

\section{Centralized network Management}

In real applications, a centralized network system is hard to implement due to the complexity of network management. In this management scenario, all secondary users are connected to the spectrum broker. As a result, it is necessary for any cognitive node that intends to transmit using any subchannel to get prior approval from the spectrum broker. This process is performed using the hierarchal multi-tier management shown in Fig. 5.

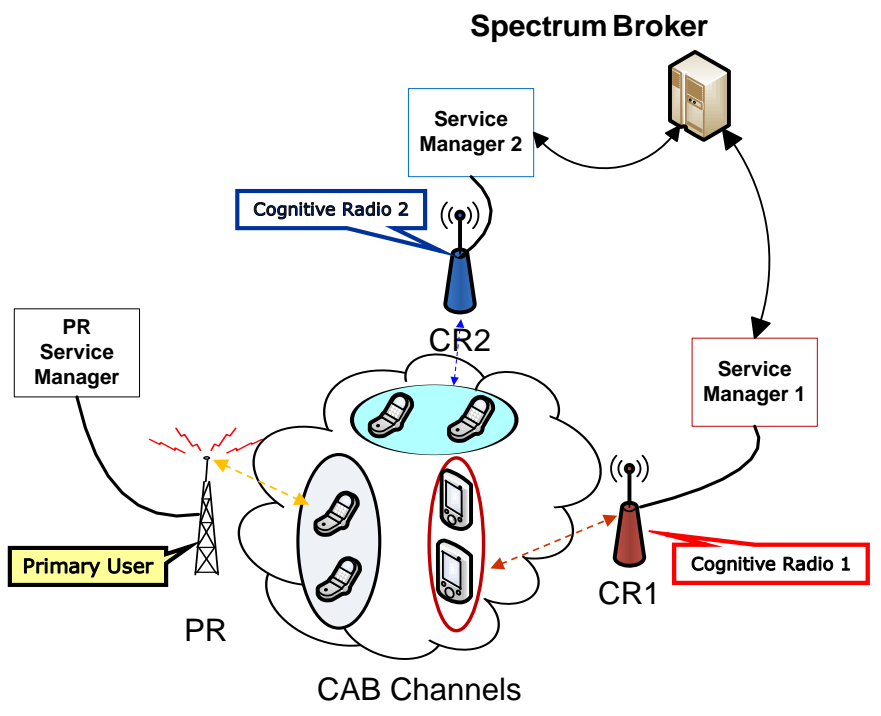

Fig. 5. Centralized network management solution.

A request for transmission is initially passed by a cognitive femtocell to the service manager (SM) of the service operator and then to the spectrum broker. The spectrum broker will then undertakes the necessary arrangements to allocate the usage time for multi-users intending to share the available spectrum. The spectrum broker generates the backhaul link, which is necessary for intermediate fair sharing of the available time among secondary users. While this seems to be an effortless way to share resources, it is actually very important to reduce the service time consumed in looking for free subchannels. Also, such a system may be the best to avoid interference between cognitive users themselves, and between cognitive and primary users as well.

We consider the performance of a cognitive network that has 
multiple cognitive users coexisting with primary users in one system model similar to [18]. The connection requests in Fig. 5 are set to follow an independent Poisson model of users $x$ in the cognitive network. With no collision in the medium access control (MAC) layer, all cognitive radio (CR) users will share the available channels with the primary network with priority given to the first arrived user. The channel capacity available for the cognitive communications is given in (8), and connections on the subchannels are shared as in (1) and (2).

The task of the spectrum broker is to allocate the spectrum between different service managers (SMs). This is performed by dividing the coordinated spectrum band (CAB) into $k$ non-overlapping blocks and assigning $S_{i}$ set of subchannels to different $m^{\text {th }}$ SMs:

$$
S_{m, i} \subset C A B, S_{m, i} \cap S_{k, i}=0, \forall m, k, i
$$

The frequency band slicing is then:

$$
S=\left(S_{1}, \ldots, S_{m}\right), \forall m
$$

where

$$
S_{m}=\left(S_{m, 1}, \ldots, S_{m, k}\right), \quad \forall m, k
$$

Assuming that the spectrum block is distributed in frequency range $(b ; e)$, where $b$ and $e$ represent spectrum boundaries. Then, let $l_{m ; i}$ denote the size of the allocated spectrum block, i.e., $l_{m ; i}=b_{m ; i}-e_{m ; i}$. To avoid interference, spectrum block $S_{m, i}$ used by $m^{\text {th }}$ provider of certain capacity within a non-overlapping region is separated by at least a minimum guard band $s_{G}$ and fits in the CAB as [19]:

$$
s_{G} \leq b_{m+1, i}-e_{m, i}, \quad \forall m, i,(m \neq M)
$$

In this way, the minimum $\mathrm{CAB}$ size $I_{C A B}$ that needs to be available to the spectrum broker to maintain performance requirements is given by:

$$
I_{C A B} \geq \sum_{m=1}^{M} l_{m ; i}+(M-1) s_{G}, \quad \forall i
$$

The main purpose of the spectrum broker is to achieve the minimum connection blocking probability through controlled dynamic spectrum access. Therefore, it is reasonable to have low- and high-priority spectrum users. We always assign the highest priority to the first arrival user's call. The prioritization between two unlicensed cognitive users should include the minimum guard band. Hence, given $N$ number of free channels at a time period, the number of transmissions accepted from unlicensed users should not exceed a threshold $L$ of occupied channels.

To analyze the success of the spectrum broker in maintaining connections with users of various priority levels, a finite-time horizon Markov decision process (MDP) was formulated for non-stationary traffic of unlicensed users. Therefore, the average number of blocked connections can be truncated to an integer given by [14]:

$$
\begin{aligned}
& P_{\text {block }}=\frac{1}{N !}\left(\frac{\lambda_{\mathrm{h}}}{\mu}\right)^{N-L}\left(\frac{\lambda_{\mathrm{h}+\lambda_{1}}}{\mu}\right)^{L} \\
& \quad \times\left[1+\sum_{x=1}^{L} \frac{1}{x !}\left(\frac{\lambda_{\mathrm{h}}+\lambda_{\mathrm{l}}}{\mu}\right)^{i}+\sum_{x=L+1}^{N} \frac{1}{x !}\left(\frac{\lambda_{\mathrm{h}}}{\mu}\right)^{x-L}\right]^{-\mathbf{1}},
\end{aligned}
$$

where $\lambda_{\mathrm{h}}$ and $\lambda_{1}$ are the main connection arrival rates for highand low-priority users, respectively, and $\mu$ is the mean connection holding time during call approval.

Then, the network throughput $\vartheta$ can be identified as:

$$
\vartheta=\sum_{x=1}^{X} a_{x} v_{x}\left(1-P_{\text {block }_{x}}\right),
$$

where $a_{x}$ is the network load, and $v_{x}$ is the total number of cells in the site when the number of cognitive users is $X$.

\section{DiSTRIBUTED NETWORK MANAGEMENT}

In this system management model, the femtocells act as the access points for the cognitive system, and share the available spectrum opportunities based on individual and group negotiations. For the case study shown in Fig. $6, \mathrm{CR}_{1}$ and $\mathrm{CR}_{2}$ can talk to each other using the common spectrum control channel (CSCC), and then can exchange information and knowledge without the need to approach the spectrum broker.

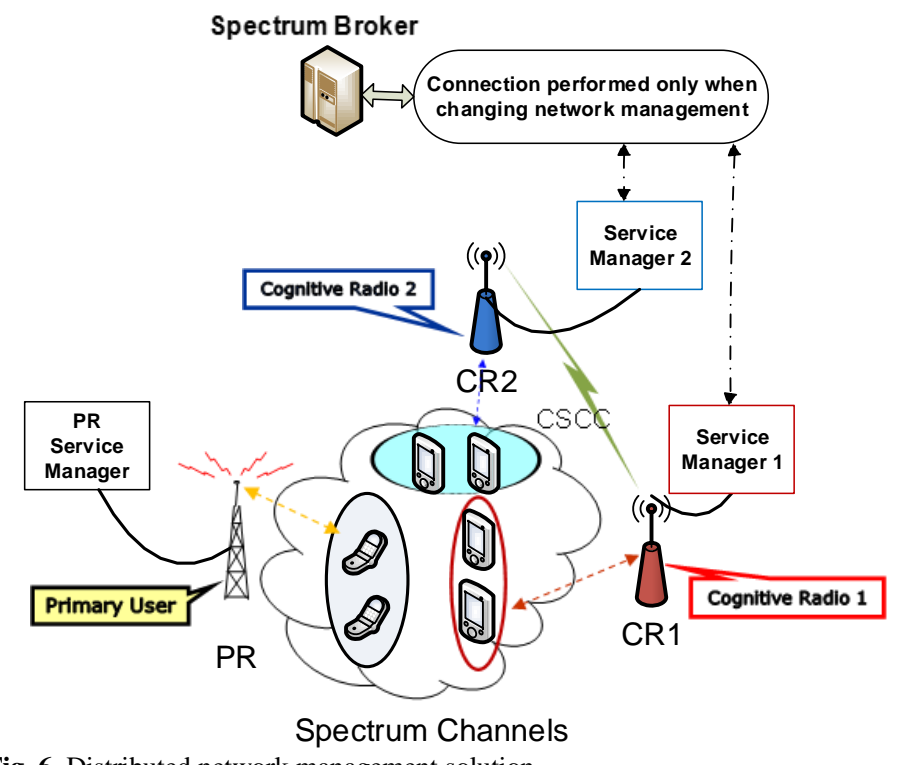

Fig. 6. Distributed network management solution.

Upon negotiations, one of those CRs will be the master and the other will be the slave. The master CR will be governing all other secondary radios and it will be the most senior user of the channel band. Therefore, $\mathrm{CR}_{1}$, along with other $\mathrm{CRs}$, manages spectrum access activations while the master should pass all updates to the spectrum broker. However, the spectrum broker is able to contact the service managers at any time to interrupt communications, whenever it determines that it is necessary to change the network management. In order to formulate a realistic system model for the performance of the distributed system, we use the IEEE802.11e technique as an approach to 
examine the performance of the distributed management.

We focus on the channel access for the femtocells. Therefore, we assume that a cognitive femtocell can transmit at any time without exceeding the cognitive channel capacity in (8). In order to evaluate the performance of the distributed cognitive femtocells, we use the 802.11a PHY characteristics to derive and capture the blocked connections and throughput. We start by analyzing the blocked connections using the 802.11e immediate chunk Ack mechanism on controlled channel access-transmission opportunities (Polled-TXOP) [20]. Fig. 7 depicts a successful case of transmission using immediate Chunk Ack policy when a polled station transmits 8 MAC protocol data unit (MPDU) frames as a chunk and a Chunk Ack Request, at time the recipient responds to a Chunk Ack Request with a Chunk Ack frame. If error information of the Chunk Ack indicates that all MAC protocol data units (MPDUs) of the block are transmitted correctly, it is counted as a successful chuck transmission [21]. This method is used to create the performance measurement probes that are distributed in various 802.11e cognitive nodes of the developed simulator that is presented in Section VIII.

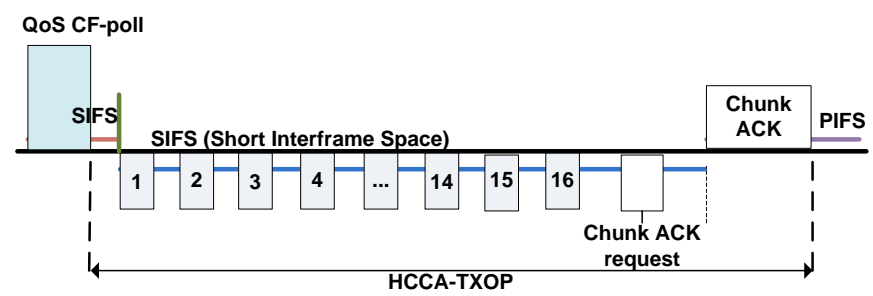

Fig. 7. Successful chunk transmission in 802.11e using chunk ACK.

In order to use the scarce spectrum as efficiently as possible, we assume that a chuck of $\sigma_{w}^{\prime}$ octet data consisting of $W$ frames is to be transmitted using PHY mode 5 over a set of subchannels $S_{i}$. Let $g$ be the average SNR per symbol at the receiver. The size of the MAC header of the data, the Chunk Ack Request, the chunk Ack, and the CF-poll are 36, 24, 152, and 36 octets, respectively. To transmit a frame with $o_{w}$ octets data over the IEEE 802.11a PHY using PHY $Б=7$, the transmission durations of the data and the Chunk Ack Request frames are:

$$
\begin{aligned}
T_{\text {data }}\left(\sigma_{w}^{\prime}, \mathrm{B}\right) & =\rho+\sigma+\left[\frac{16+8 \times\left(36+\sigma_{w}^{\prime}\right)+7}{N_{c b p s}(\mathrm{~B})}\right] \times \varphi \\
& =16 \mu s+4 \mu s+\left[\frac{304+\left(8 \times 0_{w}^{\prime}\right)+6}{192}\right] \times 4 \mu s
\end{aligned}
$$

In (17), the number 16 represents the number of bits in the frame, 8 is the number of bits per symbol, $\left(36+o_{w}^{\prime}\right)$ is the length of the data, and 7 is the number of 802.11 PHY mode 5 . This is also applicable for the following equations (18)-(20) except for the length of the data, which is different from one frame to the other.

$$
T_{b a r}(\text { Б })=\rho+\sigma+\left[\frac{16+(8 \times 24)+7}{N_{c b p s}(\mathrm{~B})}\right] \times \varphi
$$

Similarly, the transmission durations for the Block Ack and the CF-poll frames using PHY mode $\mathrm{B}$ are:

$$
\begin{gathered}
T_{b a}(\text { Б })=\rho+\sigma+\left[\frac{16+(8 \times 152)+7}{N_{c b p s}(\mathrm{~B})}\right] \times \varphi \\
T_{\text {cfpoll }}(\text { Б })=\rho+\sigma+\left[\frac{16+(8 \times 36)+7}{N_{c b p s}(\mathrm{~B})}\right] \times \varphi
\end{gathered}
$$

where $\rho$ is the required time for the 802.11e physical layer convergence protocol, and $\sigma$ is the time for prepares/parses data units transmitted/received using various 802.11e media access techniques.

Let $N_{\text {burst }}$ and $N_{M P D U}$ denote the number of burst MAC service data units (MSDUs) and the number of MAC protocol data units (MPDUs) per one MSDU, respectively. Accordingly, the number of the transmitted MPDUs per one chunk is given by $N_{\text {burst(1) }}=N_{\text {burst }} \times N_{M P D U}$, assuming that there is the same transmitted MSDUs as the MPDUs. If the CF-poll frame is delivered without error, then the probability of a successful chunk transmission of $y^{\text {th }}$ attempt to transmit $N_{\text {burst(i) }}$ MSDUs is given by [22]:

$$
\begin{gathered}
P_{g, t r n s(y)}(\sigma, g, \mathrm{~b})=\prod_{w=1}^{N_{\text {burst }(y)}}\left[1-P_{e, \text { data }}\left(\sigma_{w}^{\prime}, g_{w}, \mathrm{~b}_{w}\right)\right] \\
\times\left[1-P_{e, b a r}(g, \mathrm{~b})\right] \times\left[1-P_{e, b a}(g, \mathrm{~b})\right]
\end{gathered}
$$

where $N_{\text {burst (y) }}$ is the number of MPDUs that the transmitter retransmits after transmitting a block consisting of $N_{\text {burst }}(y)$ MPDUs at the $y^{\text {th }}$ transmission. $N_{\text {burst(y) }}$ can be calculated by [22]:

$N_{\text {burst }(y)}\left(\sigma, g\right.$, Б) $=\gamma\left[\frac{\sum_{w=1}^{N_{\text {burst }(y-1)} P_{e, \text { data }(w)}(\sigma, g, \text { Б })}}{1-P_{g, \operatorname{trans}(y-1)}(l, g, m)}\right]$,

where $\gamma[z]$ is a round function that maps $z$ to the closest integer. $P_{e, \text { data }}(\sigma, g$, Б $), P_{e, b a r}(g, m)$, and $P_{e, b a}(g, m)$ are the error probability of the data, the chunk Ack request, and chunk Ack frame, respectively.

The upper bound for the packet error probability with g-octet packet long for PHY mode 5 is identified using the binary convolutional coding and the hard-decision Viterbi decoding. This bound is given by:

$$
P_{e}^{(\mathrm{E})}(g) \leq 1-\left[1-P_{u}^{(\mathrm{b})}(g)\right]^{8 \rho},
$$

where the union bound $P_{u}^{(\mathrm{B})}(g)$ of the first-event error probability is given by the sum of the pairwise error probabilities. Thus, we obtain the union bound [23].

$$
P_{u}^{(\mathrm{B})}(g)=\sum_{\partial=\partial_{\text {free }}}^{\infty} a_{\partial} \cdot P_{\partial}(g)
$$

where $\partial_{\text {free }}$ is the free distance of the convolutional code 
selected in the PHY mode Б, $a_{\nabla}$ is the total number of error events of weight $\partial$, and $P_{\partial}(g)$ is the probability that an incorrect path at distance $\partial$ from the correct path will be chosen by the Viterbi decoder.

Therefore, the packet error probabilities of each frame can be calculated using [24]:

$$
\begin{aligned}
& P_{e, \text { data }}\left(0, g, \text { Б) }=1-\left[1-P_{e}^{1}(3, g)\right] \times\left[1-P_{e}^{(\mathrm{b})}(38.75+\alpha, g)\right]\right. \\
& P_{e, b a r}(g, \mathrm{~b})=1-\left[1-P_{e}^{1}(3, g)\right] \times\left[1-P_{e}^{(\mathrm{b})}(26.75, g)\right] \\
& P_{e, b a}(g, \mathrm{~b})=1-\left[1-P_{e}^{1}(3, g)\right] \times\left[1-P_{e}^{(\mathrm{b})}(154.75, g)\right]
\end{aligned}
$$

The probability of a successful transmission within the retry limit ${ }^{n}$ max can be calculated by:

$$
P_{\text {success }}\left(\sigma^{\prime}, g, \mathrm{~b}\right)=1-\prod_{y=1}^{\mathrm{n}} \max \left[1-P_{g, \operatorname{trans}(y)}\left(\sigma^{\prime}, g, \mathrm{E}\right)\right]
$$

Therefore, the probability of blocked connections for wireless channel condition $S_{i}$ can be given as:

$$
P_{\text {block }}=1-P_{\text {success }}(\sigma, g, \text { Б) }
$$

Assuming SIFS time of $16 \mu \mathrm{s}$, the throughput can be calculated as:

$\vartheta=\frac{8 \times \sigma^{\prime} \times N_{\text {burst }}}{T_{\text {cfpoll }}+N_{\text {burst }}+T_{\text {data }}+T_{\text {bar }}+T_{b a}+16 \mu s \times\left(N_{\text {burst }}+2\right)}$

Equations (29) and (30) are used to generate a probe to measure the probability of blockage and throughput figures at the $802.11 \mathrm{e}$ nodes. These figures, together with their replicate values that are obtained in the previous sections of the conventional and the centralized system models, are coupled to decide which management model can be used according to the time available for cognitive communications. The criterion for choosing between these management scenarios is proposed in the following section.

\section{ADAPTIVE NETWORK MANAGEMENT}

The decision to adapt between various network managements (conventional, centralized, and distributed) in response to the changes in wireless resources is determined by the spectrum broker. Such a decision will allow creating an adaptive network that adapts its architecture to attain the best performance subject to the sensing information that was obtained by the probes designed in Sections IV, V, and VI. The decision for adaptation is taken by measuring the response time of transmission actions performed by each network management model individually. The main concern here is to improve the access to transmission opportunities by adapting between different management systems. We start by identifying the response time necessary to perform any transmission interface using our studied two-tier network management, as follows:

$$
\mathrm{T}_{r}=\mathrm{t}_{S P}+\mathrm{t}_{a}+\mathrm{t}_{N}
$$

where $t_{S P}$ is the computation time required for decision making at the spectrum broker, $t_{a}$ is the time spent between the mobile user arrival at the femtocell units and the spectrum manager updates, and $\mathrm{t}_{N}$ is the network delay.

Fig. 8 shows the sequence of operations during the estimation of response time phase. The spectrum broker needs to construct a request message as many as 3 times, where 3 is the number of service managers of the studied network management systems, whereas a service manager only needs to process one round of evaluations for all scenarios.

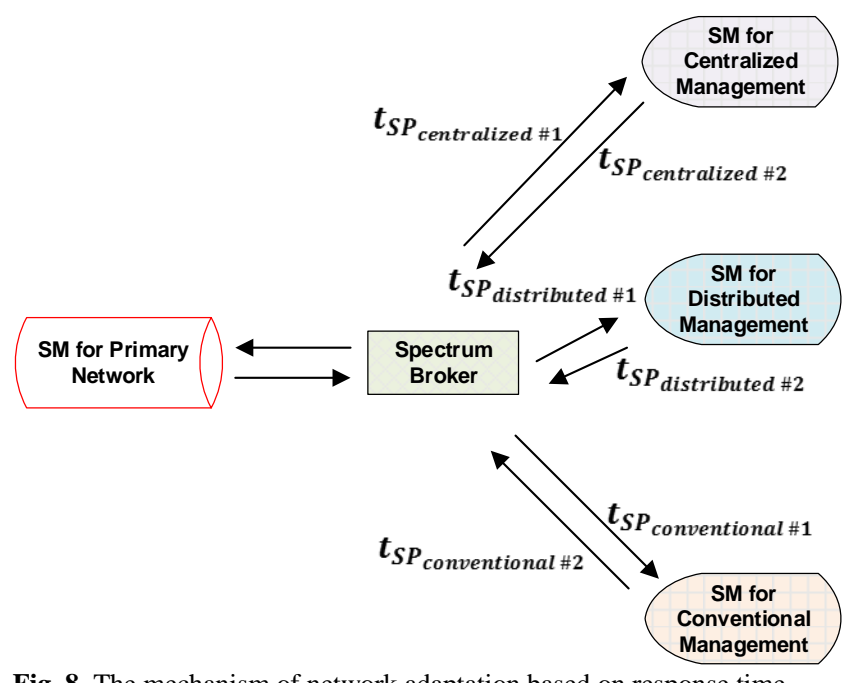

Fig. 8. The mechanism of network adaptation based on response time.

We propose to incorporate the fairness of distributing the available channels between femtocells, which was listed in Section III-A with the response time criterion of the system management. The criterion for a spectrum broker to decide upon network management adaptation is given in Table II.

In this way, the spectrum broker decides upon which network management system to operate. The choice will be for centralized management when there is at least one link that its not assigned to any subchannel. This results in a very long processing time, while the spectrum broker waits to assign the link. Using the centralized management, the spectrum broker

TABLE II

CRITERION FOR ADOPTING BETWEEN VARIOUS NETWORK MANAGEMENTS

\begin{tabular}{|l|l|l|}
\hline \multicolumn{1}{|c}{ Decision } & Response Time & Connectivity of Channels \\
\hline Centralized & $\mathrm{T}_{r} \geq 1$ & Unfair, $\left|S_{i}\right|=0$ \\
\hline Distributed & $\mathrm{T}_{r} \leq 1$ & Justified, $\left|S_{i}\right| \geq 1$ \\
\hline Conventional & $\mathrm{T}_{r}=1$ & Fair, $\left|S_{i}\right|=1$ \\
\hline
\end{tabular}

reduces the response time and assigns resources efficiently. Oppositely, the spectrum broker decides to use distributed management to maintain connectivity with users who are assigned a similar number of channels at a short response time. Finally, the spectrum broker chooses to connect users using a macrocell base station when there are large numbers of 
channels available to a lower number of users, as there is no need to go through the complexity of the macro-to-femto system.

\section{Simulation ANd Evaluation}

\section{A. Simulation Setup}

The studied cognitive network's architectures of distributed and centralized management are evaluated with respect to the conventional macro-management model using designed OPNET models. The choice to use this tool is based on the fact that examining the performance of large-scale networks is a complex challenge that needs to be solved with a very powerful computing processing system. The OPNET is capable of simulating complex heterogeneous networks of a multiple number of nodes because of its capability to mimic real-time network operations [25]. The mathematical models for channel selection and for network management's selection are coded and incorporated within the functions of the chosen simulator. The simulations incorporated cognitive radio network management models with primary network model to create a variable cognitive channel capacity as in (8). Different users are assigned to the available subchannels of the two-tier network model. The network parameters for the designed simulations are shown in Table III.

Developing different profiles of primary user activities to control the time space allocated for cognitive network created the dynamic spectrum environment. A mobile ad hoc network (MANET) gateway is used as the primary base station. This gateway achieves point-to-point communications with its users. Two MANET mobile stations are set to work as the primary end users. IEEE 802.11 devices operating with a listen-before-talk spectrum access dynamically change the operation frequencies, and dynamically control their transmission power. Thus, IEEE $802.11 \mathrm{e}$ is used to simulate the cognitive radio networks, since this 802.11 model supports the enhanced distributed channel access (EDCA) protocol. This protocol enables the $802.11 \mathrm{e}$ stations to access the spectrum in an independent and distributed manner [26].

In order to investigate the performance of the conventional, centralized, and distributed management systems, new OPNET probe functions are created and coupled with the standard models of 802.11e technology. Each conventional and centralized system uses (15) to generate the probe that measures the probability of blocked connections. In the centralized system, femtocells are set to request the spectrum broker's approval before any transmission takes place. Therefore, a new probe function is created using (16) to evaluate the throughput of the cognitive system centrally. The service and delay times are typical evaluations being obtained together with the blockage and throughput calculations by the standard system probes. Oppositely, performance evaluation is very challenging in the distributed system, as individual MAC functions are created according to the performance analysis functions of (29) and (30). These probes are coupled altogether to provide the aggregated performance characteristics of the system. The three management system models are operated in one project scenario of different free spectrum availability profiles.

The channel capacity in (8) was used to identify the maximum amount of data a network may transmit for each of the simulation iterations in order to avoid congestion and packet loss. As the main focus of this paper is to identify the architectural performance of the various cognitive network managements, it was important to evaluate the capacity of each

TABLE III

SIMULATION PARAMETER VALUES

\begin{tabular}{|c|c|}
\hline Parameter & Value \\
\hline Cell layout & $\begin{array}{l}\text { Sectors: } 1 \text { macrocell, } 2 \\
\text { femtocells, and } 14 \text { primary units }\end{array}$ \\
\hline Users active per sector & 2 \\
\hline Minimum distance to BS & 35 meters \\
\hline Propagation model & Hata-large city \\
\hline \multicolumn{2}{|l|}{ Application Layer } \\
\hline HTTP specifications & HTTP 1.1 \\
\hline Page interval time (sec) & Exponential $(720)$ \\
\hline Type of Service & Best effort $(0)$ \\
\hline \multicolumn{2}{|l|}{ Cognitive Femtocells } \\
\hline $\mathrm{HCF}$ & Supported \\
\hline Physical characteristics & OFDM $(802.11 \mathrm{a})$ \\
\hline Data rate & $48 \mathrm{Mbps}$ \\
\hline Maximum transmission power & $1 \mathrm{~mW}$ \\
\hline \multicolumn{2}{|l|}{ Primary Network } \\
\hline Physical characteristics & Direct sequence \\
\hline Data rate & $11 \mathrm{Mbps}$ \\
\hline Channel bandwidth & $22 \mathrm{MHz}$ \\
\hline Max. Receive lifetime & $0.5 \mathrm{secs}$ \\
\hline
\end{tabular}

of the used channels to decide upon the available free space for cognitive communications as well as avoid significant packet loss by mapping the size of the network sectors and the amount of transmitted power to the available spectrum. We used the animation feature in OPNET to validate and monitor packets for each channel and used the exported simulator reports to track all channels. System setup specified the capacity the traffic transmitted per second for 122 discrete iterations of simulation as shown in Table III.

\section{B. Experimental Results}

The system performance was evaluated as a function of the load/demand and the spectrum availability. One particular interest of performance is the network capacity. In this measure, the total network throughput is measured in order to evaluate the implications of changing the various network management models using different profiles of spectrum occupation. The network performance is compared for the three case study scenarios of conventional, centralized, and distributed network management. The collected results from various simulation iterations are average values, obtained as a function of free time that is available for cognitive communications. Therefore, the probe results are allocated at 
the $y$-axis and the free time for cognitive communication is represented at the $\mathrm{x}$-axis of the following graphs.

The traffic is not a steady-state phenomenon, and the spectrum availability fluctuates dynamically in the simulated cognitive network model. Fig. 9 shows that the centralized network management system scenario provides higher throughput most of the time, followed by the distributed and conventional cognitive radio network management schemes. Specifically, the distributed model shows a higher throughput for (10-20) \% of the free time available for cognitive communications. The explanation for this is that self-managed femtocells can easily access short free transmission intervals with no need to obtain prior assignment from the spectrum broker. This ensures that the connectivity of users to the available channels $\left|S_{i}\right| \geq 1 \forall i \epsilon\left\{1, \ldots,\left|E_{t}\right|\right\}$ a justified assignment. As the transmission opportunity increases, it can be seen that the centralized system throughput is the highest among other cognitive network management systems for most of the free time. When there is more than one link that is not assigned to any subchannel, the system is unfair and the spectrum broker acts as the prominent manager that assigns channels to the users in operation.

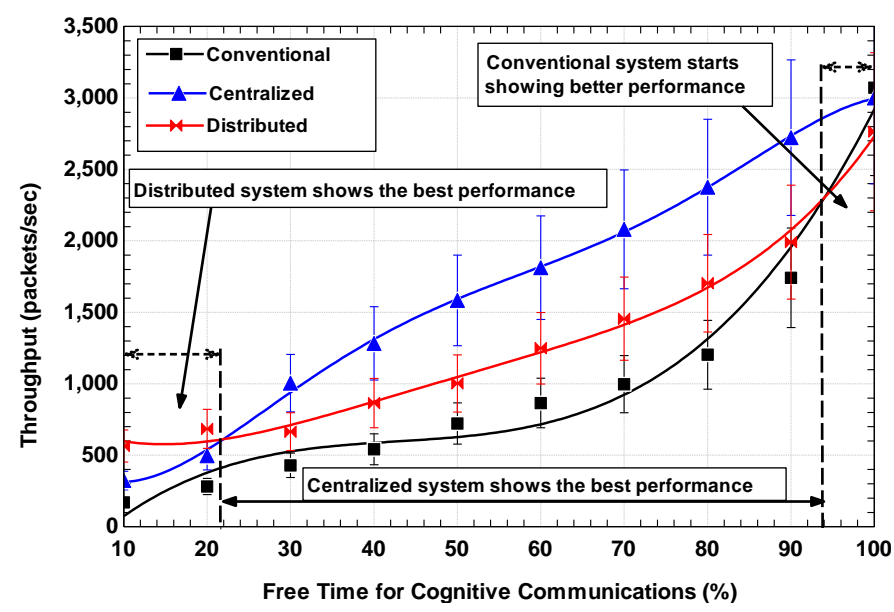

Fig. 9. Cognitive throughput vs. free time for cognitive communications.

The conventional model of network management system shows the lowest throughput for most of the time because a multi-access system of small cells can explore transmission opportunities much better than the macro-system model. However, the macro-system model shows a higher performance that exceeds both the centralized and distributed systems for more than $90 \%$ of the free time available for cognitive communication. This is the fair channel assignment case, where approaching users through macrocell has less complexity than scattering the limited resources between multi-numbers of femtocells.

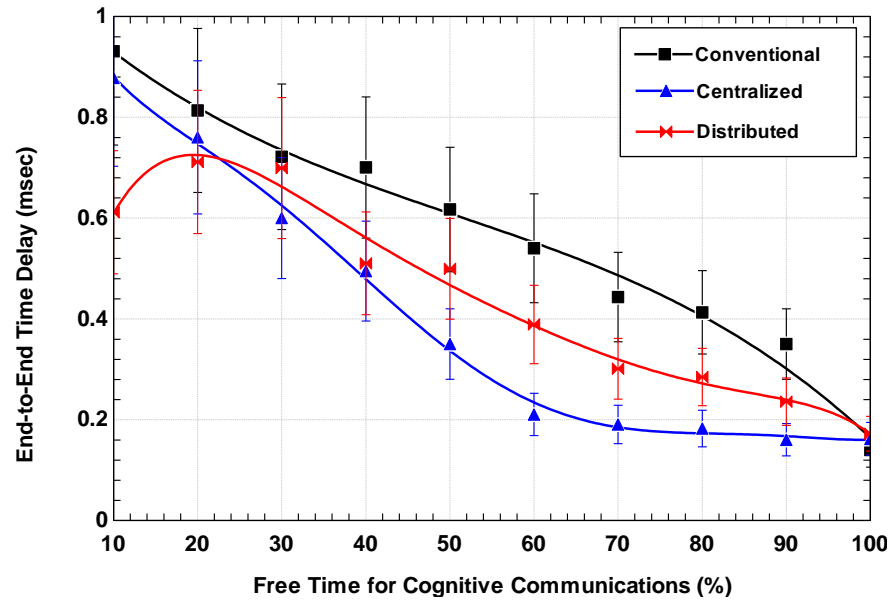

Fig. 10. Cognitive end-to-end time delay vs. free time for cognitive communications.

Fig. 10 compares the end-to-end time delays for the three studied cognitive radio network management systems. The $\mathrm{x}$-axis shows the free time that is allocated to the cognitive communications. All of the three systems above give a similar bound on the end-to-end delay time, which is close to that when almost all of the time is allocated to the cognitive radio network at the end of the simulation. The conventional macro-system model followed by the distributed and centralized systems for most of the simulation time incurs major time delays. The end-to-end delay decreases since the resources available for cognitive systems increase. However, the decline rates are quite different at the beginning and at the end of the simulation.

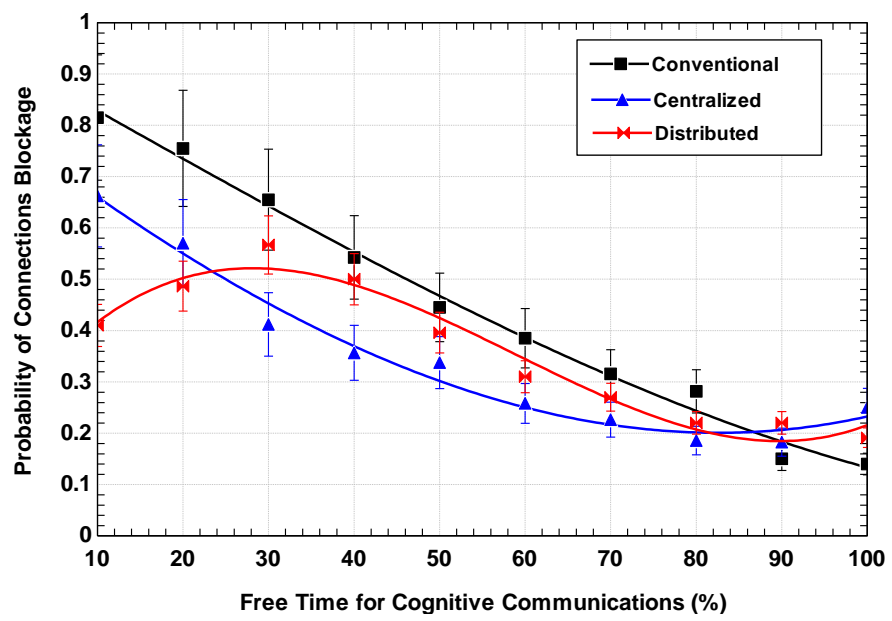

Fig. 11. Probability of connections blockage vs. free time for cognitive communications.

Fig. 11 depicts that the probability of blocked connections is maximum when there are few transmission opportunities available for cognitive communications with the maximum values at $10 \%$ on available free time. Similarly, the performance has the same differences between various cognitive radio network management systems. This means that macro-only system networks have the maximum blocked connections probability due to the fact that smaller cells can approach end users more efficiently in very dynamic wireless 
environments. The performance is gradually improved at the end of the simulation due to the growing amount of available free time for cognitive communications.

Response time is an important factor to determine the success of the cognitive network selection system in adapting network architectures in response to any changes in the spectrum availability. Therefore, we measure the response delay for all simulated management scenarios, as in Fig. 12, to explore the efficiency of different cases in service adaptation time prior to actual transmissions.

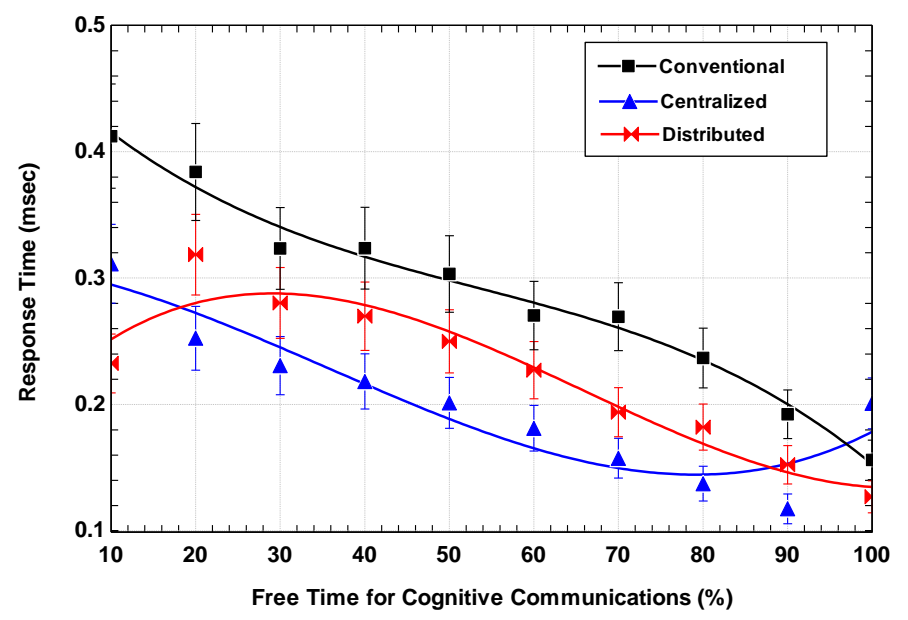

Fig. 12. Cognitive response time vs. free time for cognitive communications.

Clearly, the response time declines in all management cases when there are more transmission intervals available for cognitive users. This means that there is more flexibility in adapting system architectures and domains at shorter times for transmissions to end users when the primary users are less effective. It can be seen that the centralized management model shows a better performance most of the time.

Overall, the simulations show the superiority of the centralized management system over the distributed and conventional cognitive radio network systems for almost $80 \%$ of the time available for cognitive communications. This has been proven through a significant increase of throughput and a reduction in the end-to-end time delay for cognitive radio network employing small cells of femtocells in the cognitive access system. These results will help to manage the adaptation between various network management schemes. This can be performed using the mechanism proposed in Section VII through evaluating the response time provided by the SMs to the spectrum broker.

\section{CONCLUSIONS}

Integrating macro and femtocells through a proper network management system allows more flexibility for the allocation and use of the scarce spectrum in the cognitive radio networks. This paper investigated and evaluated various system managements for cognitive network planning: conventional, centralized, and distributed. A cognitive selection framework is developed to decide upon the appropriate management system for different network operational situations. Models for channel assignment and cognitive channels capacity were discussed to develop a large-sized cognitive radio network that incorporates femtocells. Performance analyses were done to examine various network management systems that incorporate femtocells and compare them with the traditional macrocell system. Results show that a hybrid system supported by a macrocell and centralized/distributed femtocells showed an improved performance in intelligently adapting the resource allocation in response to wireless environment changes. A comprehensive study for management systems in cognitive radio network is proposed for future mobile operators and research communities for further development.

\section{REFERENCES}

[1] S. Liu, L. Lazos, and M. Krunz, "Cluster-Based Control Channel Allocation in Opportunistic Cognitive Radio Networks," IEEE Transactions on Mobile Computing, vol. 11, no. 10, pp. 1436 1449, Oct. 2012.

[2] B. Wang and K. J. Ray Liu, "Advances in Cognitive Radio Networks: A Survey," IEEE Journal of Selected Topics in Signal Processing, vol. 5, no. 1, pp. 5 - 23, Feb. 2011.

[3] Cisco, Introduction to eTOM, white paper, 2009.

[4] W. C. Cheung, T. Q. S. Quek, and M. Kountouris, "Throughput Optimization, Spectrum Allocation, and Access Control in Two-Tier Femtocell Networks," IEEE Journal on Selected Areas in Communications, vol. 30, no. 3, pp. 561 - 574, Apr. 2012.

[5] L. Li, C. Xu, and M. Tao, "Resource Allocation in Open Access OFDMA Femtocell Networks," IEEE Wireless Communications Letters, vol. 1, no. 6, pp. 625 - 628, Dec. 2012.

[6] S.-M. Cheng, W.-C. Ao, F.-M. Tseng, and K.-C. Chen, "Design and Analysis of Downlink Spectrum Sharing in Two-Tier Cognitive Femto Networks," IEEE Transaction on Vehicular Technology, vol. 61, no. 5, pp. 2194 - 2207, Jun. 2012.

[7] F. Jin, R. Zhang, and L. Hanzo, "Fractional Frequency Reuse Aided Twin-Layer Femtocell Networks: Analysis, Design and Optimization," IEEE Transactions on Communications, vol. 61, no. 5, pp. 2074 - 2085, May 2013.

[8] H. S. Dhillon, R. K. Ganti, F. Baccelli, and J. G. Andrews, "Modeling and Analysis of K-Tier Downlink Heterogeneous Cellular Networks," IEEE Journal on Selected Areas in Communications, vol. 30, no. 3, pp. 550 - 560, Apr. 2012.

[9] J. Xiang, Y. Zhang, T. Skeie, and L. Xie , "Downlink Spectrum Sharing for Cognitive Radio Femtocell Networks," IEEE Systems Journal, vol. 4 , no. 4, pp. 524 - 534, Dec. 2010.

[10] B. Han, W. Wang, Y. Li, and M. Peng, "Investigation of Interference Margin for the Co-existence of Macrocell and Femtocell in Orthogonal Frequency Division Multiple Access Systems," IEEE Systems Journal, vol. 7, no. 1, pp. 59 - 67, Mar. 2013.

[11] Federal communication commission (2002) spectrum policy task force, Rep.ET Docket no. 02-135.

[12] A. Anand and R. Chandramouli, "Resource Allocation in Cognitive Radio Networks" in Y. Xiao and F. Hu (Eds.), Cognitive Radio Networks, CRC Press, Jan. 2009.

[13] Q. Ni and C. Zarakovitis, "Nash Bargaining Game Theoretic Scheduling for Joint Channel and Power Allocation in Cognitive Radio System," IEEE Journal on Selected Areas in Communications, vol. 30, no. 1, pp. 70 - 81, Jan. 2012.

[14] E. Ain, D. Niyato, and Z. Han, Dynamic Spectrum Access and Management in Cognitive Radio Networks, Cambridge University Press, 2009.

[15] H.-L. Fu, P. Lin, and Y.-B. Lin, "Reducing Signaling Overhead for Femtocell/Macrocell Networks," IEEE Transactions on Mobile Computing, vol. 12, no. 8, pp. 1587 - 1597, Aug. 2013. 
[16] A. Al-Dulaimi, A. Anpalagan, and J. Cosmas, "Spectral Efficiency of Neighbourhood Femtocell Networks," WWRF\#31 meeting, Vancouver, Canada, 22-24 Oct. 2013.

[17] S. Al-Rubaye, A. Al-Dulaimi, and J. Cosmas, "Cognitive Femtocell, Future Wireless Networks for Indoor Applications," IEEE Vehicular Technology Magazine, vol. 6, no. 1, pp. 44 - 51, Mar. 2011.

[18] B. Wang, Z. Ji, and K. J. R. Liu, "Primary-Prioritized Markov Approach for Dynamic Spectrum Access," in Proc. of IEEE Intern. Symp. on New Frontiers in Dynamic Spectrum Access Networks (DySPAN2007), pp. 507 - 515.

[19] L. Kovács and A. Vidács, "Dynamic Spectrum Allocation using Regional Spectrum Brokers," in Dynamic Communications Management (pp. 6-1 - 6-14). Meeting Proceedings RTO-MP-IST-062, Paper 6. Neuilly-sur-Seine, France: RTO, 2006.

[20] S. Rashwand and J. Misic, "IEEE 802.11e EDCA Under Bursty Traffic-How Much TXOP Can Improve Performance," IEEE Transactions on Vehicular Technology, vol. 60, no. 3, pp. 1099 1115, Mar. 2011.

[21] K. Zhou and A. Kunisa, "Cross-Layer Optimization for A-MPDU with HT-immediate Block Acknowledgement in Non-Competitive Channel Environment," IEEE Consumer Comm. and Networking Conf. (CCNC), Las Vegas, USA, pp. 971 - 972, 9 - 12 Jan. 2011

[22] Y. Xu, J. C. Lui, and D. Chiu, "Improving Energy Efficiency via Probabilistic Rate Combination in S02.11 Multi-Rate Wireless Networks," Ad Hoc Networks, vol. 7, pp. 1370 - 1385, 2009.

[23] L. H. C. Lee, "New Rate-Compatible Punctured Convolutional Codes for Viterbi Decoding," IEEE Transaction on Communication, vol. 42, no. 12, pp. 3073 - 3079, Dec. 1994.

[24] S. Haykin, Digital Communications, $3^{\text {rd }}$ ed. John Wiley \& Sons, Inc., 1994.

[25] J. Prokkola, "OPNET - Network simulator," Tutorial, VTT Technical Research Center of Finland, 2006.

[26] A. Al-Dulaimi, H. S. Al-Raweshidy, J. Cosmas, J. Loo, "Cognitive Mesh Networks," IEEE Vehicular Technology Magazine, vol. 5, no. 3, pp. 54 - 60, Sept. 2010. 